\title{
Different effects of double-pulseTMS of the posterior parietal cortex on reflexive and voluntary saccades
}

\section{Zoi Kapoula*, Qing Yang, Norman Sabbah and Marine Vernet}

IRIS Group, Centre d'Etudes SensoriMotrices, UMR8194, CNRS, Service d'ophtalmologie, Hôpital Européen Georges Pompidou, Paris, France

\section{Edited by:}

Shuhei Yamaguchi, Shimane

University, Japan

\section{Reviewed by:}

Yoshikazu Ugawa, Fukushima Medical

University, Japan

Tatsuya Mima, Kyoto University

School of Medicine, Japan

*Correspondence:

Zoi Kapoula, IRIS Group, UMR 8194,

CNRS, Service d'ophtalmologie,

Hôpital Européen Georges Pompidou,

20, rue Leblanc, 75015 Paris, France.

e-mail:zoi.kapoula@egp.aphp.fr
Gap and overlap tasks are widely used to promote automatic versus controlled saccades. This study examines the hypothesis that the right posterior parietal cortex (PPC) is differently involved in the two tasks. Twelve healthy students participated in the experiment. We used double-pulse transcranial magnetic stimulation (dTMS) on the right PPC, the first pulse delivered at the target onset and the second 65 or 80 ms later. Each subject performed several blocks of gap or overlap task with or without dTMS. Eye movements were recorded with an Eyelink device. The results show an increase of latency of saccades after dTMS of the right PPC for both tasks but for different time windows $(0-80 \mathrm{~ms}$ for the gap task, 0-65 ms for the overlap task). Moreover, for rightward saccades the coefficient of variation of latency increased in the gap task but decreased in the overlap task. Finally, in the gap task and for leftward saccades only, dTMS at 0-80 ms decreased the amplitude and the speed of saccades. Although the study is preliminary and needs further investigation in detail, the results support the hypothesis that the right PPC is involved differently in the initiation of the saccades for the two tasks: in the gap task the PPC controls saccade triggering while in the overlap task it could be a relay to the Frontal Eye Fields which is known to control voluntary saccades, e.g., memory-guided and perhaps the controlled saccades in the overlap task The results have theoretical and clinical significance as gap-overlap tasks are easy to perform even in advanced age and in patients with neurodegenerative diseases.

Keywords: latency, gap effect, amplitude, speed, variability

\section{INTRODUCTION}

Gap and overlap tasks have been widely used for studying saccades. In the gap task the fixation point switches off and the peripheral target appears later, typically $200 \mathrm{~ms}$; in the overlap task the peripheral target appears while the fixation point is still on. Latencies are 40-110 ms shorter in the gap task. This effect called "gap effect" (Saslow, 1967) is believed to be related to advance movement preparation due to release of the oculomotor fixation in the gap task (Rolfs and Vitu, 2007). It is believed that in the gap task, saccade initiation is triggered by the posterior parietal cortex (PPC; Pierrot-Deseilligny et al., 1995). We hypothesize that saccades in the overlap task are triggered by the frontal eye field (FEF), similarly to more voluntary saccades such as memory-guided saccades (Wipfli et al., 2001). The hypothesis of different initiation mechanisms in the gap and overlap tasks has been supported by our behavioral study (Vernet et al., 2009). Vernet et al. (2009) studied gap-overlap tasks in pure or mixed blocks for young and middle age subjects. They reported a cost of interleaving the gap and overlap trials, i.e., an increase of latency relative to no mixing (pure blocks); the mixing effect was observed for middle age adults only. Even though age-specific, the cost due to mixing indicates different mechanisms for saccade triggering in gap versus overlap tasks. The present study aims to test further the hypothesis of different mechanisms in the gap and overlap tasks, with the use of double-pulse transcranial magnetic stimulation (dTMS). Prior studies using single-pulse TMS and the gap task only, showed that TMS of the PPC increases latency (Kapoula et al., 2001, 2005).
The dTMS produces facilitatory or inhibitory effects depending on the interstimulus interval (ISI, see Siebner and Rothwell, 2003). The few other studies which used dTMS concern memory-guided saccades (saccades made upon a go signal to a target flashed a few seconds earlier): Wipfli et al. (2001) showed the decrease of latency of contralateral memory-guided saccades after dTMS of the FEF with the ISI of $50 \mathrm{~ms}$ (first pulse on "go" signal, the second $50 \mathrm{~ms}$ later). Nyffeler et al. (2005) also showed such facilitation after dTMS of the PPC but with ISI of $80 \mathrm{~ms}$. In our study the first pulse over the PPC is delivered at the onset of the saccade target and the second 65 or $80 \mathrm{~ms}$ later. We expect inhibitory effects of the dTMS, i.e., latency increase at least in the gap task, as this area is believed to participate in the triggering of saccades. For the overlap task we expect either facilitatory effects or still inhibitory effects but at different time windows than for the gap task. Note that expectation for inhibitory effect is not in contradiction with the study of Nyffeler et al. (2005) showing latency decrease. In the memory-guided task used by those authors dTMS was delivered on the PPC upon extinction of the central dot and $80 \mathrm{~ms}$ later. In an overlap task we use here both the central and peripheral targets are on at the times of dTMS, requiring visual attention engagement. Thus, although we hypothesize a flow of signal processing from PPC to FEF for the overlap task, the mechanisms of attention and fixation control involved might not be the same as for memory-guided saccades.

Our prior studies with single-pulse TMS in a gap condition have shown the importance of PPC on saccade triggering, particularly 
of the right PPC. Indeed, TMS of the right PPC delivered 80$100 \mathrm{~ms}$ after target onset increases saccade latency in all directions. Therefore in the present study, as a first step, we concentrated on the right PPC which is known to be greatly involved in saccade control. The timing parameters for the first and second pulse were chosen so as to interfere with the triggering process in the gap task. Based on prior studies with the gap task we expected an effect when dTMS is delivered at 0 and $80 \mathrm{~ms}$ after target onset; we thought opposing it to an earlier window (0-65 ms) for which TMS could be less effective. We used the same time windows for the overlap task. If the PPC is involved the same way as in the gap task, then we should expect the same effects of dTMS for the same time windows. We aimed to rule out such prediction.

Another novelty of our study is related to the effect of dTMS on the variability of latency of saccades. Gilchrist et al. (2009) proposed several hypotheses to explain the naturally occurring variability of saccade latency, e.g., fluctuation of the quality of processing of target location or decision mechanism in triggering or of the fixation control. Carpenter (2004) introduced a decisionmaking model (Later model) consistent with neurophysiological data, that explains the variability and correctly predicts the effects of altered expectations. If, as we hypothesize, the PPC is involved differently in the gap and overlap tasks, the effects of dTMS on variability in latency might also be different. Finally, although our study concerns primarily the initiation mechanisms it also examined the effect of dTMS on the amplitude and the speed of saccades, and this for the two tasks (gap, overlap).

\section{MATERIALS AND METHODS \\ SUBJECTS}

Twelve healthy adult subjects, four females and eight males, participated in this experiment. Their ages ranged from 18 to 23 years (mean $21 \pm 1.5$ ). All subjects had normal or correctedto-normal vision. Binocular vision was assessed with the TNO test of stereoacuity; individual scores were normal, $60^{\prime \prime}$ of arc or better except for two subjects $\left(120^{\prime \prime}\right)$. Each subject gave informed consent to participate in the study.

The eye movement investigation adhered to the tenets of the Declaration of Helsinki and was approved by the local human experimentation committee, CPP Il de France II (No: 07035), Hospital Necker in Paris. Written consent was obtained from all subjects after the nature of the examination had been explained.

\section{TMS LOCALIZATION}

A MagStim 200 magnetic stimulator was used. Its maximum stimulator output was 2.2 T (model 200 MagStim, Withland, Wales, $\mathrm{UK}$ ); the coil has a figure-of-eight (each wing $70 \mathrm{~mm}$ diameter) allowing focal stimulation. The time of increase of the pulse was $5 \mu \mathrm{s}$, the decay lasted $160 \mu$ s. A click occurred simultaneously with the pulse.

To define motor thresholds, the intersection of the wings of the coil was applied on the motor hand area and the intensity of the stimulator was increased until it reached a value for which visible jerks of contralateral hand muscles occurred. For our group of subjects, the motor threshold ranked from 40 to $60 \%$ of total stimulator output. During PPC stimulation, the intensity was between 50 and $66 \%$, depending on the subject. Such values, above the motor threshold, did not cause blinks.

The right PPC was stimulated by placing the coil $3 \mathrm{~cm}$ posteriorly and $3 \mathrm{~cm}$ laterally to the vertex, tangentially to the skull, in order to obtain a well focalized stimulation of this area. The coil was placed on the scalp with its handle oriented backward and $45^{\circ}$ rightward (for stimulation of the right PPC) relative to the midline. Similar procedures for localization, coil placement, and stimulation capacity have been used by many studies (Muri et al., 1996; Kapoula et al., 2001, 2004). In the dTMS blocks, doublepulse stimulation was applied on the right PPC. TMS was delivered twice, on the onset of target for $(0 \mathrm{~ms})$ and 65 or $80 \mathrm{~ms}$ after target appearance. We will refer to these intervals as ISI. For the blocks without TMS, stimulation was also delivered at the same time windows, but the coil was placed $30 \mathrm{~cm}$ over the head of the subject and oriented toward the ceiling, in order to provide the same auditory input in both conditions (dTMS/no dTMS). A second coil, unlinked to the magnetic stimulator was placed over the subject head, in order to conserve the same somato-sensory clues as during the real stimulation. A similar control condition has been used by others (Terao et al., 1998; Yang and Kapoula, 2004).

\section{STIMULI/VISUAL DISPLAY}

The visual display was composed by three white luminous dots (angular size $0.2^{\circ}$ ), presented on a black computer screen placed at $57 \mathrm{~cm}$ from the subject (the required convergence angle was about $6^{\circ}$ ). One of these three dots was at the center of the screen; two were at an eccentricity of $\pm 10^{\circ}$ horizontally.

The subject was comfortably seated in an adapted chair with his chin on a chin rest. The subject viewed binocularly.

\section{EYE MOVEMENT RECORDING}

Horizontal and vertical eye movements were recorded binocularly with the EyeLink II device. Each channel was sampled at $250 \mathrm{~Hz}$. The system has a resolution of $0.025^{\circ}$.

\section{GAP AND OVERLAP TASKS}

Each trial started by lighting a fixation dot at the center. The fixation dot stayed on for a period of $2.5 \mathrm{~s}$. In the gap task, there was a time interval of $200 \mathrm{~ms}$ between the offset of the fixation dot and the onset of the saccade target dot at the periphery dot (Figure 1A). The target dot was kept on for $1.5 \mathrm{~s}$. In the overlap task, the fixation dot remained illuminated for $200 \mathrm{~ms}$ after the target dot appeared (Figure 1B). The target stayed on also for another $1.5 \mathrm{~s}$. Subjects were required to make a horizontal saccade to the target dot as rapidly and accurately as possible. A black period of $2.5 \mathrm{~s}$ separated trials. Subjects were instructed to use this period for blinks. The total mean length of each trial was about $6.7 \mathrm{~s}$. In each block, targets at $10^{\circ}$, left or right, were interleaved randomly at equal rates. Each block contained 32 trials and lasted about 4 min (16 trials for saccades to the left and 16 trials for saccades to the right interleaved randomly). Each subject performed six blocks, four blocks with dTMS (ISI $65 \mathrm{~ms}$ gap; ISI $80 \mathrm{~ms}$ gap; ISI $65 \mathrm{~ms}$ overlap; ISI 80 ms overlap) and two blocks without TMS in the gap and overlap conditions. The order of blocks was counter balanced over subjects. For each dTMS condition dTMS was delivered in $95 \%$ of the trials. A few trials without TMS were introduced to reduce expectancy effects. 


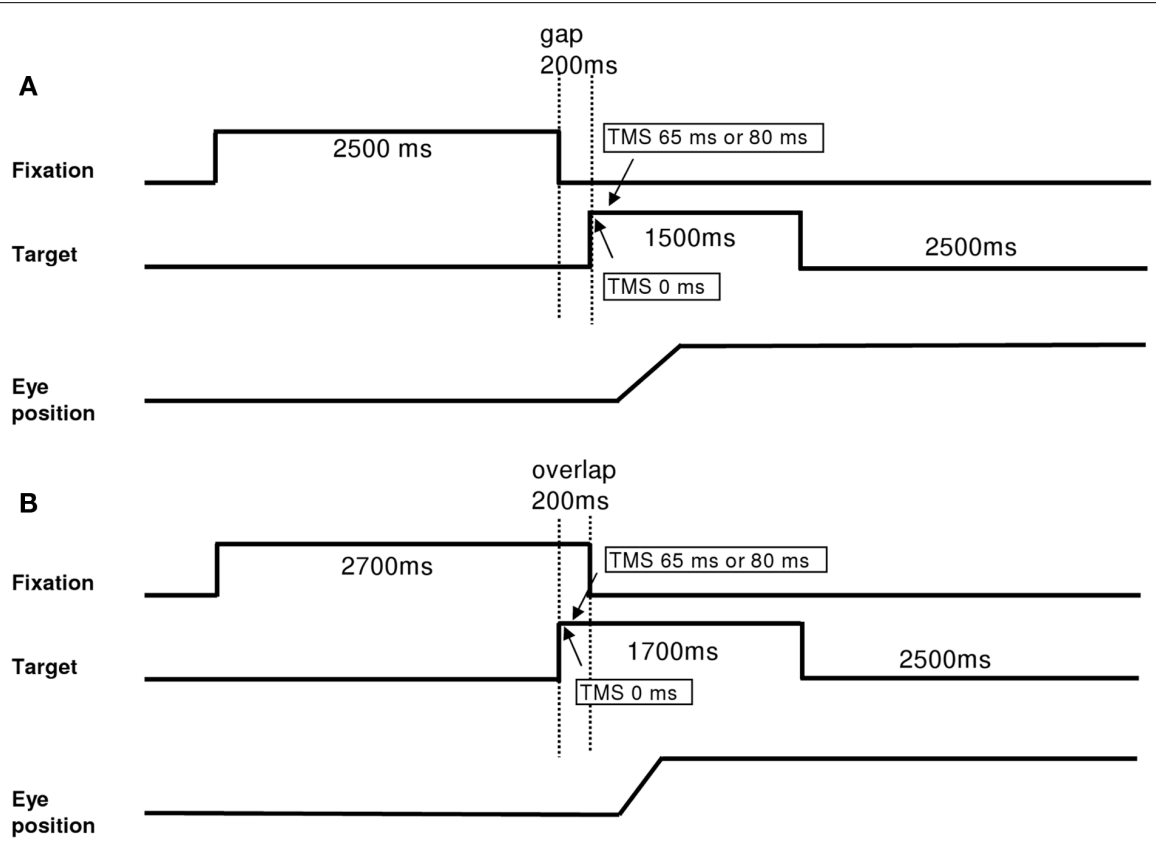

FIGURE 1 | Experimental tasks: gap (A) and overlap (B). Latency started when the target appeared. For blocks with dTMS, the first stimulation was done with the onset of the target $(0 \mathrm{~ms})$ and the second stimulation 65 or $80 \mathrm{~ms}$ after, in both tasks.

A calibration sequence was performed at the beginning; the target made the following predictive sequence: center, $10^{\circ}$ to left, center, $10^{\circ}$ to right, center; the target stayed at each location for $2 \mathrm{~s}$. From these recordings we extracted calibration factors.

\section{DATA ANALYSIS}

Calibration factors of each eye were extracted from the saccades recorded during the calibration task. A calibration was run on the horizontal signals with a linear function to fit the calibration data. From the two individual calibrated horizontal eye position signals, we derived the horizontal conjugate signal (mean of the two horizontal eyes position). Example of saccade from one subject is shown in Figure 2.

The onset and offset of horizontal saccades was defined as the time when the eye speed of the conjugate signal exceeded and dropped below $10 \%$ of the maximum velocity, respectively. The onset and the offset of the saccade are noted " $i$ " and " $p$ " and the maximum velocity " $v$ " in Figure 2. These criteria are standard and used in several other studies (Yang and Kapoula, 2004; Yang et al., 2006; Vernet et al., 2009). The automatic placement of the markers by the computer was verified by visual inspection of the individual eye movement traces. From these markers, we measured the latency (ms) of eyes movements, the amplitude (deg) and mean speed (deg/s) of the saccade. The mean speed was the amplitude divided by the duration. The coefficient of variation (CV) of latency is the SD of latency divided by the mean latency multiplied by 100 (Peltsch et al., 2011; Kapoula et al., 2010). The $\mathrm{CV}$ was measured for the other parameters (amplitude, speed). The percentage of express latency is the number of saccades with latency values between 80 and $120 \mathrm{~ms}$, divided by the total number of saccades, and multiplied by 100 (Kapoula et al., 2004, 2005; Yang and Kapoula, 2006; Yang et al., 2006).

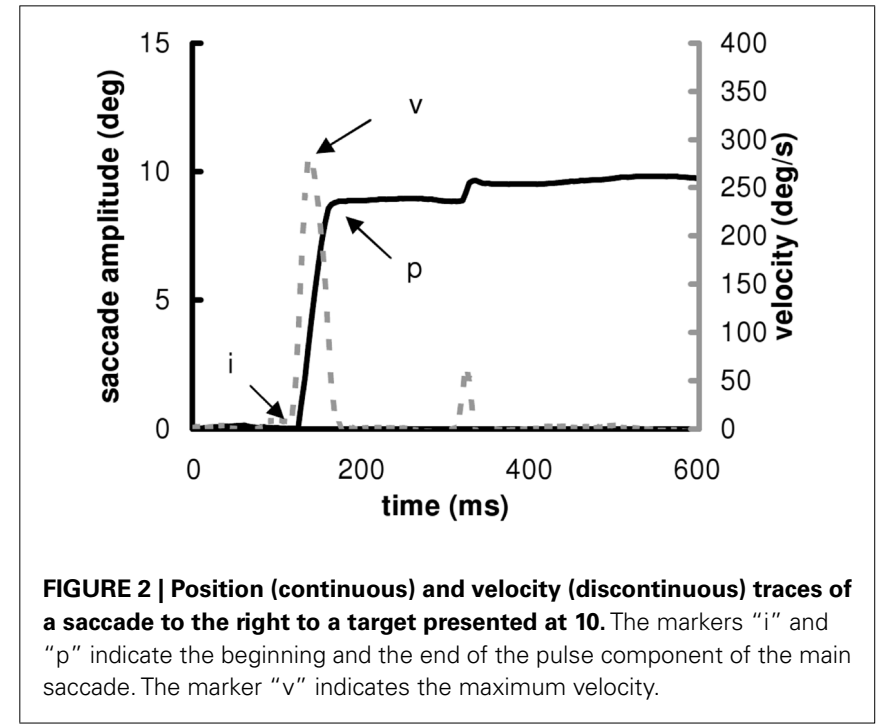

Saccades in the wrong direction, saccades contaminated by blinks, saccades with latency shorter than $80 \mathrm{~ms}$, and saccades with amplitude below $50 \%$ or above $150 \%$ of required amplitude were excluded from the analysis. In total, $4 \%$ of the movements were rejected, the most frequent reason being the blinks.

\section{STATISTICAL EVALUATION OF MEANS}

A three-way ANOVA was applied on individual means for each saccade parameter (latency, speed, amplitude) with three inter-subjects factors: type of dTMS (no dTMS; dTMS ISI 65 ms; dTMS ISI $80 \mathrm{~ms}$ ), saccade direction (left; right), type of task (gap; overlap). Given the large number of conditions tested and the 
relatively small number of subjects, local comparisons were made with post hoc tests. The Tukey's honestly significant difference (HSD) post hoc test (significance level $P<0.05$ ) was used applied on any two means for significance or tendency; individual data were inspected showing same tendency for the majority of subjects in case of significant post hoc effects. The non-parametric, Friedman and Wilcoxon tests were applied on the CV of latencies, amplitude, and speed.

\section{RESULTS}

\section{DIFFERENT EFFECT OF dTMS ON THE LATENCY IN THE GAP AND OVERLAP TASKS}

Firstly, there was a main effect of the gap/overlap task (ANOVA, $F_{1,11}=733$; $P<0.001$; Figure 3 ). The magnitude of this effect was $99 \mathrm{~ms}$, i.e., latencies are longer in the overlap task than in the gap task (all conditions merged, no dTMS, dTMS, or direction); there was no interaction between task and dTMS condition $\left(F_{2,22}=1.21, P=0.32\right)$. Thus, the gap effect was stable (Figure 1B) for all conditions (no dTMS, dTMS ISI $65 \mathrm{~ms}$, dTMS ISI $80 \mathrm{~ms}$, $\left.F_{2,22}=2.61, P=0.10\right)$, and for saccade direction $\left(F_{1.11}=0.62\right.$, $P=0.49$ ), There is no significant interaction between dTMS condition and saccade direction.

Given the large number of conditions tested we proceeded to local analysis with the Tukey's HSD test. Group mean latencies
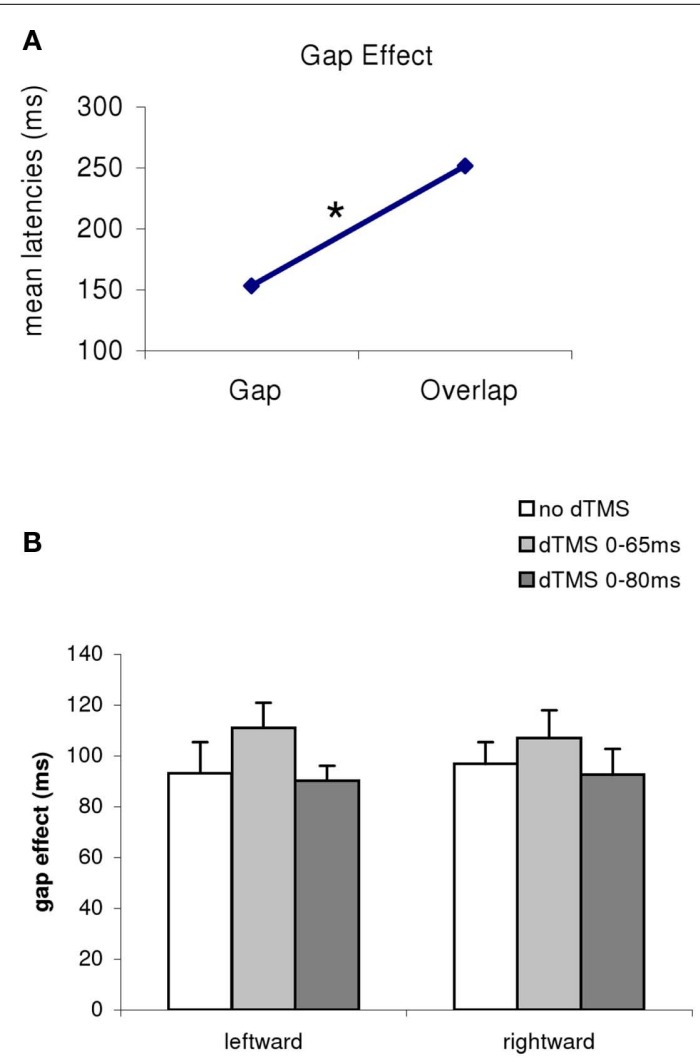

FIGURE 3 | (A) Mean latencies of the group according to the task (gap/overlap), and (B) gap effect for all conditions merged [(B), dTMS, saccade direction] The asterisk indicates a main effect of the gap/overlap task. The magnitude of this effect is $99 \mathrm{~ms}$ longer in the overlap task. for each time window of dTMS, for each saccade direction and for each task are represented in Figure 4. For saccades directed to both sides for the gap task (Figure 4A) there was a significant increase $(P<0.01)$ of the mean latency by the dTMS of ISI $80 \mathrm{~ms}$; the effect was $15 \mathrm{~ms}$ for saccades to left and $12 \mathrm{~ms}$ for saccades to right. For the overlap task, a significant increase $(P<0.001)$ was found for another dTMS of ISI $65 \mathrm{~ms}$ and only for saccades to the left (Figure 4B); the effect was $22 \mathrm{~ms}$. For individual data (see Figure A1 in Appendix).

\section{NO dTMS EFFECT ON THE RATE OF EXPRESS SACCADES}

As expected, the Gap task induced express saccades with latencies between 80 and $120 \mathrm{~ms}$, the group mean rate of express latencies was $32.0 \%$ (range, $0-100 \%$ ) for saccades to the left and $22.1 \%$ (range, $0-100 \%$ ) for saccades to the right. There was no significant effect of the dTMS on these express saccades latencies whatever the condition. Thus the effects on mean latencies reported above were not mediated by changes in the percentage of express latencies.

\section{DIFFERENT EFFECTS OF dTMS ON THE VARIABILITY OF LATENCY IN GAP AND OVERLAP TASKS}

Figure 5 shows the CV in latencies for the group; data are shown for no dTMS and for each dTMS condition in the gap and overlap tasks. For the gap task, dTMS with an ISI of $80 \mathrm{~ms}$ leads to a statistically significant $(Z=2.20 ; P<0.05)$ increase of latency variability of saccades directed to the right (Figure 5A). On the opposite, in the overlap task dTMS with an ISI of $65 \mathrm{~ms}$ causes statistically significant decrease $(Z=2.04 ; P<0.05)$ for saccades directed to the right (Figure 5B). So, dTMS has a different effect on latency variability for the gap and overlap tasks (increase versus decrease) and occurs at different time windows. Note that the baseline variability for saccades to right tended to be larger but it was not significantly different from that for saccades to the left $(Z=1.26, P=0.21)$.

\section{SPECIFIC EFFECTS OF dTMS ON THE MEAN SPEED AND AMPLITUDE OF SACCADES IN THE GAP TASK}

We studied all other parameters, the mean speed, and the amplitude for each task of saccade, with and without dTMS (Figures 6 and 7). The three-way ANOVA showed no main effect either of the task (gap/overlap; speed $F_{1,9}=0.02, P=0.88$, amplitude $F_{1,9}=0.001, P=0.98$ ), or of the dTMS (speed $F_{2,18}=2.64$, $P=0.10$; amplitude $\left.F_{2,18}=2.64, P=0.10\right)$, or of the saccade direction (speed $F_{1,9}=0.03, P=0.86$; amplitude $F_{1,9}=4.39$, $P=0.07)$. No significant interactions were observed either between any of the factors (all $P>0.05$ ). Post hoc analysis shows a statistically significant decrease of the mean amplitude $(P<0.01$, the effect was $\left.0.9^{\circ}\right)$; also a statistically significant decrease of the mean speed after dTMS with an ISI of $80 \mathrm{~ms}(P<0.05$, the effect was $22^{\circ} / \mathrm{s}$ ); this effect is highly specific and is present only for the gap task and for saccades directed to the left (Figures 6A and 7A); There is no significant effect of dTMS on the variability of either of these two parameters.

Thus, dTMS in the gap task at ISI of $80 \mathrm{~ms}$ has a rather massif influence, affecting latency but also amplitude and speed of leftward saccades. 

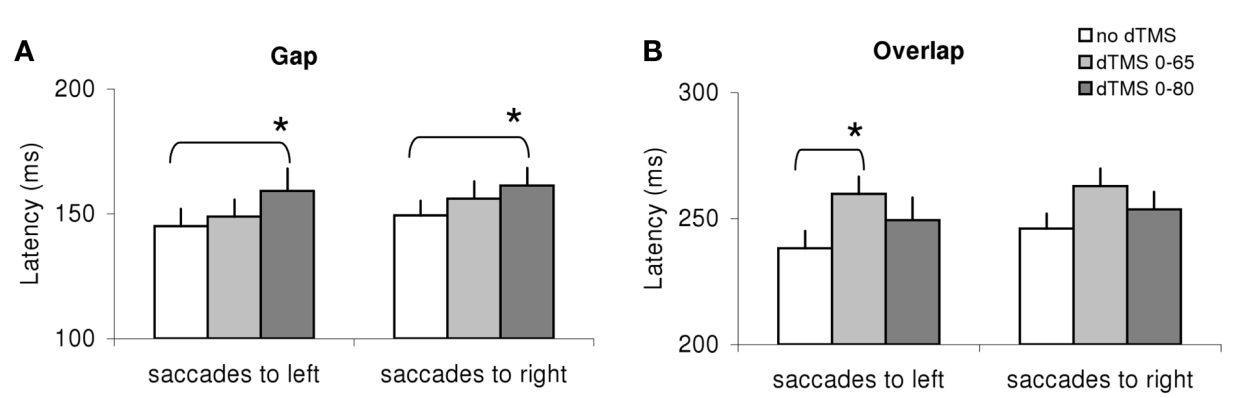

FIGURE 4 | Group mean latencies with SE for saccades to left or right in different dTMS conditions: no dTMS, dTMS 0-65 ms, dTMS 0-80 ms under the tasks gap (A) and overlap (B). Asterisks indicate a significant effect on group mean latencies of dTMS with an ISI of 65 or 80 ms versus control no dTMS.
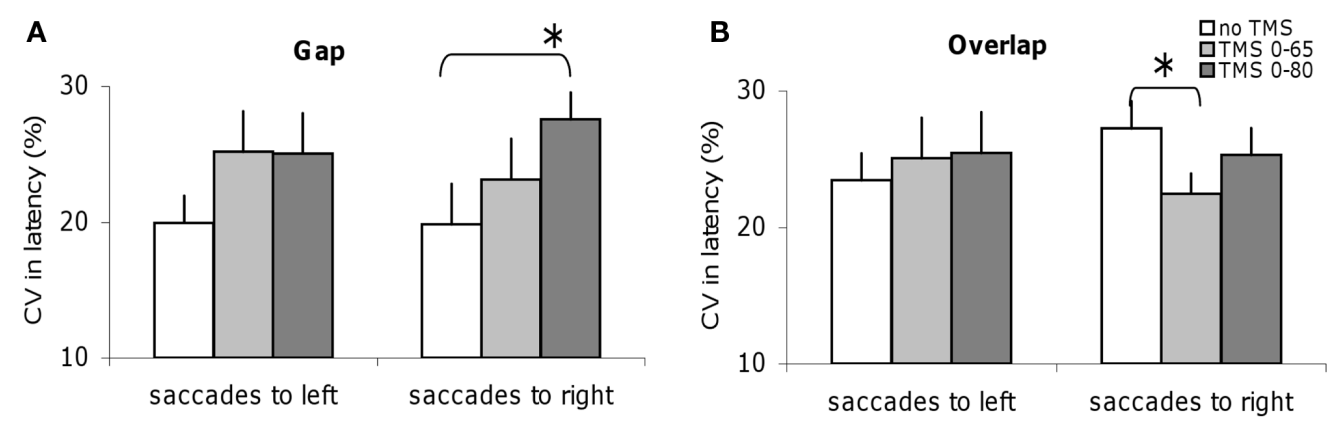

FIGURE 5 | Coefficient of variation of saccade latencies for the group subjects in different dTMS conditions: no dTMS, dTMS 0-65 ms, dTMS 0-80 ms under the tasks gap (A) and overlap (B). Asterisks indicate a significant effect on group mean values of dTMS with an ISI of 65 or 80 ms versus control no dTMS.
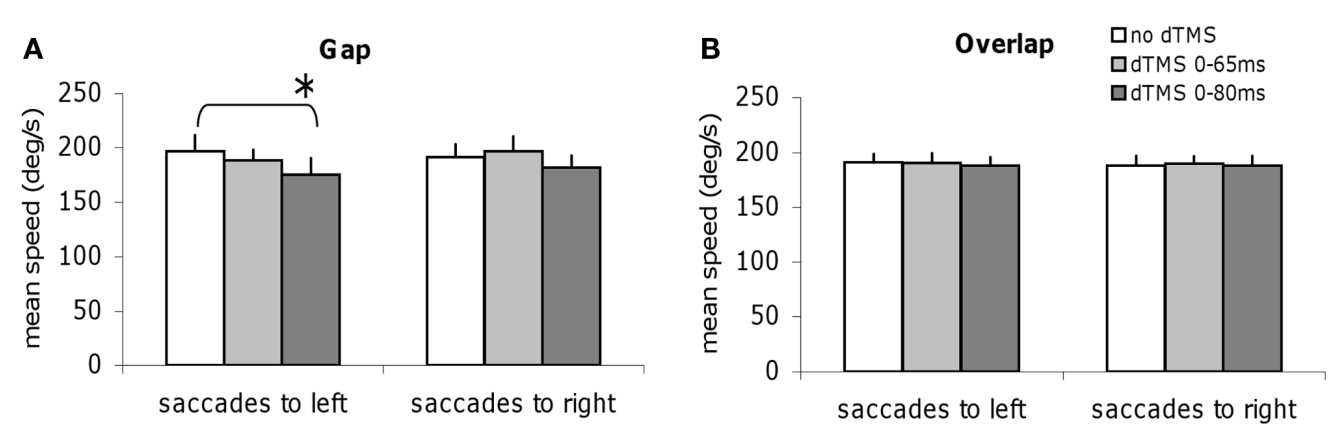

FIGURE 6 | Group mean speed with SE of saccades for saccades to left or to right in different dTMS conditions: no dTMS, dTMS 0-65 ms, dTMS $\mathbf{0 - 8 0} \mathbf{~ m s}$ under the tasks gap (A) and overlap (B). Asterisks indicate a significant effect on group mean speed of dTMS with an ISI of 65 or 80 ms versus control no dTMS.

\section{DISCUSSION}

The main findings are (i) a robust gap effect that is unaltered by the dTMS of the right PPC; (ii) different effect of the dTMS on the mean saccade latency in the gap versus overlap paradigm; (iii) no effect of dTMS on the frequency of express latency; (iv) different effect of dTMS on the variability of latency in the gap and overlap tasks; (v) specific effect on the amplitude and speed of leftward saccades in the gap task only. These results will be further discussed below.

\section{ROBUST GAP EFFECT}

A robust gap effect, namely, shorter latencies in the gap task than in the overlap task has been observed; the effect was $92-110 \mathrm{~ms}$ and was unaltered by dTMS. Similar gap effect has been found by many other studies (Schiller et al., 1987; Munoz et al., 1998; Bucci et al., 2005; Yang and Kapoula, 2006; Yang et al., 2006). This result can be explained by the release of the fixation in the gap task; competition between the two points (central versus peripheral) leads to a latency increase in the overlap task (Dorris et al., 1997; Rolfs 

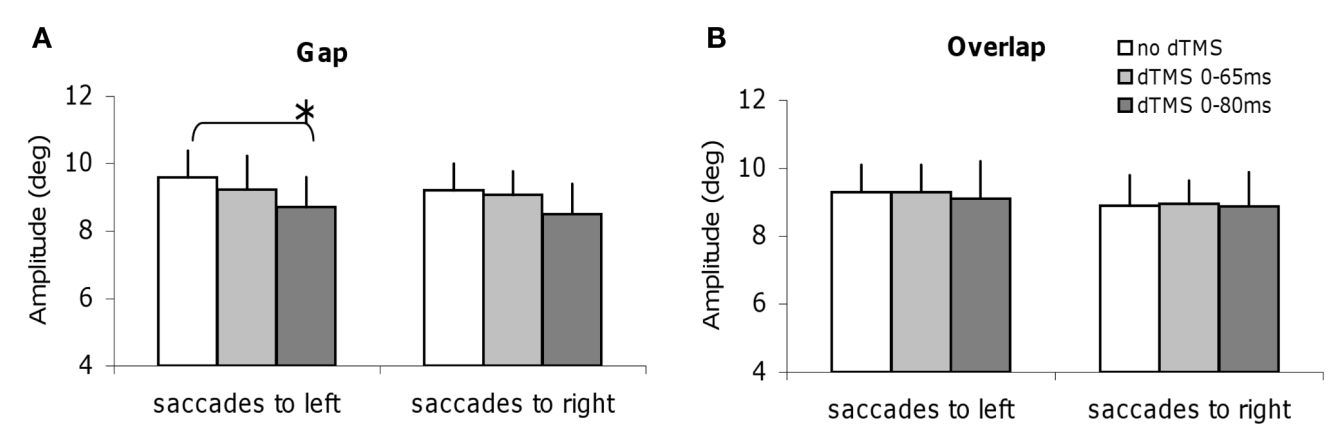

FIGURE 7 | Mean amplitude of saccades with SE for the group subjects in different dTMS conditions: no dTMS, dTMS 0-65 ms, dTMS 0-80 ms under the tasks gap (A) and overlap (B). Asterisks indicate a significant effect on group mean amplitude of dTMS with an ISI of 65 or 80 ms versus control no dTMS.

and Vitu, 2007). Advanced motor preparation in the gap task is another mechanism (Rolfs and Vitu, 2007; Vernet et al., 2009).

\section{EFFECTS OF dTMS ON MEAN LATENCY: TIME WINDOW SPECIFICITY/BILATERAL-UNILATERAL ASPECTS}

The results show latency increase after dTMS of the right PPC with an ISI of $65 \mathrm{~ms}$ in the overlap task but with an ISI of $80 \mathrm{~ms}$ in the gap task. One could question to what extent the effects on latency could be due to inter-sensory facilitation. Indeed Terao et al. (1997) showed shortening of manual reaction time with sub threshold TMS over the motor cortex; they reported similar effects with electrical stimulation of the neck or when a click sound was heard near the head. The authors suggested that much of the effect of TMS on simple manual reaction time may be ascribed to inter-sensory facilitation. In the present study we observed an increase of saccade latency which is not compatible with the idea of peripheral inter-sensory facilitation effect. Moreover our effects of latency increase are specific to certain time window (see below) and this argues against unspecific inter-sensory facilitation mechanisms. The time window specificity of the effects corroborates our initial hypothesis for different mechanisms controlling the triggering of saccades in the gap and overlap tasks. Excitatory inputs from the right PPC to the superior colliculus are believed to be responsible for the saccade triggering in the gap task, releasing the fixation system (Kapoula et al., 2004). We suggest that dTMS interferes with these excitatory inputs leading to a delay of the fixation disengagement at the level of the superior colliculus and thus to a latency increase in the gap task. In contrast, triggering of saccades in the overlap task, would involve another mechanism similar to that for memory-guided saccades, i.e., with a flow of target information passing from the PPC to the FEF from where the trigger signal is sent to the superior colliculus. Thus, the earlier effect in the overlap task supports our hypothesis that the PPC in this task and at this time window could only be a relay to the FEF; the FEF is perhaps the organizer of all controlled saccades including those from the overlap task as suggested also by Brown et al. (2004). Yet, this is still an hypothesis calling for further experiments, namely studies with dTMS of the FEF.

Considering the dTMS effect itself with different ISIs one can propose two hypothetical mechanisms. At first, it is possible that the effect of the longer ISI dTMS may be due to longer in duration stimulation, and this can discern two different time windows. Another possibility is that the dTMS can have a summation effect, so that the dTMS with shorter ISI might have stronger effect. The results exposed are compatible with the longer duration mechanism. For the gap task the longer ISI could produce longer effect in duration interfering with saccade triggering by the PPC. For the overlap task, long ISI and duration effect might be inefficient as the PPC is hypothetically only briefly involved as a relay. Further dTMS studies with different ISIs would be of interest to explore the time course for the two tasks.

It is also interesting that the effect on latency is bilateral for the gap task but contralateral (to the stimulated site) for the overlap task. The first result is compatible with studies of PierrotDeseilligny et al. (2002) who reported bilateral saccade latency increase in patients with right PPC lesions. The results are also in line with our prior single-pulse TMS studies of the PPC, showing omnidirection effects after stimulation of the right PPC (see Kapoula et al., 2004). For the overlap task, our observation of a contralateral effect only is novel and compatible with the hypothetical mechanisms we propose: in this task the contralateral PPC sends spatial information to the FEF which finally triggers the saccade.

In summary, this study shows that dTMS of the right PPC increases latency of saccades, similarly to what was reported before for single-pulse TMS (Kapoula et al., 2001, 2004). Most important, the increase of latency by dTMS is observed for different time windows for the gap and overlap tasks and with different laterality aspects. Time specificity for the gap versus overlap tasks is a novel finding providing evidence that the PPC is involved differently in the control of the saccade for the gap and overlap tasks.

\section{NO EFFECT OF dTMS ON THE RATE OF EXPRESS SACCADES}

There is no change on the rate of express saccades with or without dTMS; thus, the right PPC seems not to control the triggering of these movements. The results are in line with models of direct subcortical loops, i.e., retina-superior colliculus such as that proposed by Isa and Kobayashi (2004) controlling the triggering of express saccades. 


\section{LATENCY VARIABILITY: DIFFERENTIAL EFFECTS IN GAP AND OVERLAP TASKS}

In the gap task, latency variability increased with dTMS with ISI of $80 \mathrm{~ms}$, but decreased in the overlap task with dTMS with ISI $65 \mathrm{~ms}$. In theory, the hypothetical inter-sensory facilitation mechanisms mentioned above could reduce latency variability with or without change in the mean latency. Yet, we observed increase of variability in the gap task and decrease in the overlap task only. Such highly specific effect, again argue for a limited role, if any, of possible inter-sensory facilitation acting in the overlap task only. Rather we privilege the central differential mechanisms discussed below. In the overlap task the PPC is itself source of variability, by its interplay with the FEF for the saccade triggering.

Variability can also be interpreted in the context of stochastic models linked to decisions mechanisms (Carpenter, 2004). The reduction of variability in the overlap task could be related to the controlled nature of this task; dTMS could even intensify such controlled mode, thus, reducing variability. In the gap task, the triggering of saccades is more automatic, so, local decision mechanisms may be less active, leading to uncontrolled variability.

To our knowledge, this is the first time that dTMS effects on latency variability are reported. The differential effects on latency variability for the two tasks, are, again, in favor of the idea of differential involvement of the PPC in the control of saccades in the gap and overlap tasks.

Finally, we should discuss the direction of the effects on variability. For the gap task the variability tended to increase for both saccade directions but reached statistical significance only for the rightward saccades. The general increase of variability is compatible with the hypothetical scheme we propose for an omnidirection involvement of the right PPC in the gap task. Perhaps with more subjects leftward saccades would also show significant increase of latency variability. In the overlap task, the observation of decrease of variability for rightward saccades is more surprising. Note however, for the baseline (no dTMS condition) rightward saccades in the overlap task are the ones with the longer latency and more variability (even though the difference was not significant). Perhaps in this case, the dTMS acts as a warning signal decreasing variability.

\section{REFERENCES}

Brown, J. W., Bullock, D., and Grossberg, S. (2004). How laminar frontal cortex and basal ganglia circuits interact to control planned and reactive saccades. Neural Netw. 17, 471-510.

Bucci, M. P., Pouvreau, N., Yang, Q., and Kapoula, Z. (2005). Influence of gap and overlap paradigms on saccade latencies and vergence eye movements in seven-year-old children. Exp. Brain Res. 164, 48-57.

Carpenter, R. H. (2004). Contrast, probability, and saccadic latency; evidence for independence of detection and decision. Curr. Biol. 14, 1576-1580.

Dorris, M. C., Pare, M., and Munoz, D. P. (1997). Neuronal activity in monkey superior colliculus related to the initiation of saccadic eye movements. J. Neurosci. 17, 8566-8579.

Gilchrist, I. D., Sumner, P., Muthukumaraswamy, S., and Singh, K. (2009). "The origins of individual saccade latency variability," in 15th European Conference on Eye Movements, Southampton, 169.

Isa, T., and Kobayashi, Y. (2004). Switching between cortical and subcortical sensorimotor pathways. Prog. Brain Res. 143, 299-305.

Kapoula, Z., Isotalo, E., Muri, R. M., Bucci, M. P., and RivaudPechoux, S. (2001). Effects of transcranial magnetic stimulation of the posterior parietal cortex on saccades and vergence. Neuroreport 12, 4041-4046.

Kapoula, Z., Yang, Q., Coubard, O., Daunys, G., and Orssaud, C. (2004).

\section{EFFECTS ON AMPLITUDE AND SPEED OF LEFTWARD SACCADES IN THE GAP TASK ONLY}

The decrease of the amplitude and of the speed specifically for leftward saccades and in the gap task only after dTMS with ISI of $80 \mathrm{~ms}$ also shows that the PPC has a fundamental role in the gap task; at this specific time window it handles many parameters of the movement (latency, speed, movement amplitude); dTMS of the PPC presumably, disrupts both, temporal and spatial saccade signals. Recall, that the speed of saccades is primarily determined by the brainstem saccade generator located in the PPRF (Leigh and Zee, 2006). The effect of dTMS of the PPC on the mean speed of saccades is of interest as it indicates, that the cortical signal from PPC to the brainstem (via the superior colliculus) could also modify the speed of the saccade. This result is consistent with the idea that the TMS interferes with the function of the complete network involved in the generation of the movement (cortical, subcortical).

Once more, the specificity of the effects on the mean speed and amplitude of saccades provide evidence in favor of our main hypothesis for different control mechanisms in the gap and overlap tasks. Effects of dTMS on the speed and amplitude of saccades in the gap task are presented here for the first time. Other studies (Kapoula et al., 2004) using single-pulse TMS did not report such effects. Perhaps, using dTMS produced a more massive interference with the PPC function. Also it is interesting that this effect is seen only for leftward saccades, i.e., contralateral to the stimulated site. Further studies with TMS of the left PPC should decrease velocity of rightward saccades only, but this remains to be shown.

In conclusion, the study provides preliminary evidence for different mechanisms controlling saccades in the gap and overlap tasks. The study calls for further investigation in detail and has clinical significance. Gap and overlap paradigms are relative easy to perform without conscious effort even for advanced elderly and patients.

\section{ACKNOWLEDGMENTS}

Supported by GIS Institute of Complex Systems, Paris-Il de France, convention no: IF09-1969/R.

Transcranial magnetic stimulation of the posterior parietal cortex delays the latency of both isolated and combined vergence-saccade movements in humans. Neurosci. Lett. 360, 95-99.

Kapoula, Z., Yang, Q., Coubard, O. Daunys, G., and Orssaud, C. (2005). Role of the posterior parietal cortex in the initiation of saccades and vergence: right/left functional asymmetry. Ann. N. Y. Acad. Sci. 1039, 184-197.

Kapoula, Z., Yang, Q., Vernet, M., Dieudonné, B., Greffard, S., and Verny, M. (2010). Spread deficits in initiation, speed and accuracy of horizontal and vertical automatic saccades in dementia ith lewy bodies. Front. Neurol. 1:138. doi:10.3389/fneur.2010.00138
Leigh, R. J., and Zee, D. S. (2006). The Neurology of Eye Movement. New York: Oxford University Press.

Munoz, D. P., Broughton, J. R., Goldring, J. E., and Armstrong, I. T. (1998). Age-related performance of human subjects on saccadic eye movement tasks. Exp. Brain Res. 121, 391-400.

Muri, R. M., Vermersch, A. I., Rivaud, S., Gaymard, B., and PierrotDeseilligny, C. (1996). Effects of single-pulse transcranial magnetic stimulation over the prefrontal and posterior parietal cortices during memory-guided saccades in humans. J. Neurophysiol. 76, 2102-2106.

Nyffeler, T., Egli, A., Pflugshaupt, T., von Wartburg, R., Wurtz, P., Mosimann, U., Hess, C. W., and Muri, R. M. 
(2005). The role of the human posterior parietal cortex in memoryguided saccade execution: a doublepulse transcranial magnetic stimulation study. Eur. J. Neurosci. 22, 535-538.

Peltsch, A., Hemraj, A., Garcia, A., and Munoz, D. P. (2011). Agerelated trends in saccade characteristics among the elderly. Neurobiol. Aging 32, 669-679.

Pierrot-Deseilligny, C., Ploner, C. J., Muri, R. M., Gaymard, B., and Rivaud-Pechoux, S. (2002). Effects of cortical lesions on saccadic: eye movements in humans. Ann. N. Y. Acad. Sci. 956, 216-229.

Pierrot-Deseilligny, C., Rivaud, S., Gaymard, B., Muri, R., and Vermersch, A. I. (1995). Cortical control of saccades. Ann. Neurol. 37, 557-567.

Rolfs, M., and Vitu, F. (2007). On the limited role of target onset in the gap task: support for the motorpreparation hypothesis. J. Vis. 7, $1-20$.

Saslow, M. G. (1967). Effects of components of dispacement-step stimuli upon latency for saccadic eye movement. J. Opt. Soc. Am. 57, 1024-1029.

Schiller, P. H., Sandell, J. H., and Maunsell, J. H. (1987). The effect of frontal eye field and superior colliculus lesions on saccadic latencies in the rhesus monkey. J. Neurophysiol. 57, 1033-1049.

Siebner, H. R., and Rothwell, J. (2003). Transcranial magnetic stimulation: new insights into representational cortical plasticity. Exp. Brain Res. 148, 1-16.

Terao, Y., Fukuda, H., Ugawa, Y., Hikosaka, O., Hanajima, R., Furubayashi, T., Sakai, K., Miyauchi, S., Sasaki, Y., and Kanazawa, I. (1998). Visualization of the information flow through human oculomotor cortical regions by transcranial magnetic stimulation. J. Neurophysiol. 80, 936-946.

Terao, Y., Ugawa, Y., Suzuki, M., Sakai, K., Hanajima, R., GembaShimizu, K., and Kanazawa, I. (1997). Shortening of simple reaction time by peripheral electrical and submotor-threshold magnetic cortical stimulation. Exp. Brain Res. 115, 541-545.

Vernet, M., Yang, Q., Gruselle, M., Trams, M., and Kapoula, Z. (2009). Switching between gap and overlap pro-saccades: cost or benefit? Exp. Brain Res. 197, 49-58.

Wipfli, M., Felblinger, J., Mosimann, U. P., Hess, C. W., Schlaepfer, T. E. and Muri, R. M. (2001). Doublepulse transcranial magnetic stimulation over the frontal eye field facilitates triggering of memory-guided saccades. Eur. J. Neurosci. 14 571-575.

Yang, Q., and Kapoula, Z. (2004). TMS over the left posterior parietal cortex prolongs latency of contralateral saccades and convergence. Invest. Ophthalmol. Vis. Sci. 45, 2231-2239.

Yang, Q., and Kapoula, Z. (2006). The control of vertical saccades in aged subjects. Exp. Brain Res. 171, 67-77.

Yang, Q., Kapoula, Z., Debay, E., Coubard, O., Orssaud, C., and Samson, M. (2006). Prolongation of latency of horizontal saccades in elderly is distance and task specific. Vision Res. 46, 751-759.

Conflict of Interest Statement: The authors declare that the research was conducted in the absence of any commercial or financial relationships that could be construed as a potential conflict of interest.

Received: 17 June 2011; accepted: 26 September 2011; published online: 13 October 2011.

Citation: Kapoula Z, Yang Q, Sabbah N and Vernet $M$ (2011) Different effects of double-pulse TMS of the posterior parietal cortex on reflexive and voluntary saccades. Front. Hum. Neurosci. 5:114. doi 10.3389/fnhum.2011.00114

Copyright (C) 2011 Kapoula, Yang, Sabbah and Vernet. This is an open-acces article subject to a non-exclusive license between the authors and Frontiers Media $S A$, which permits use, distribution and reproduction in other forums, provided the original authors and source are credited and other Frontiers conditions are complied with. 


\section{APPENDIX}

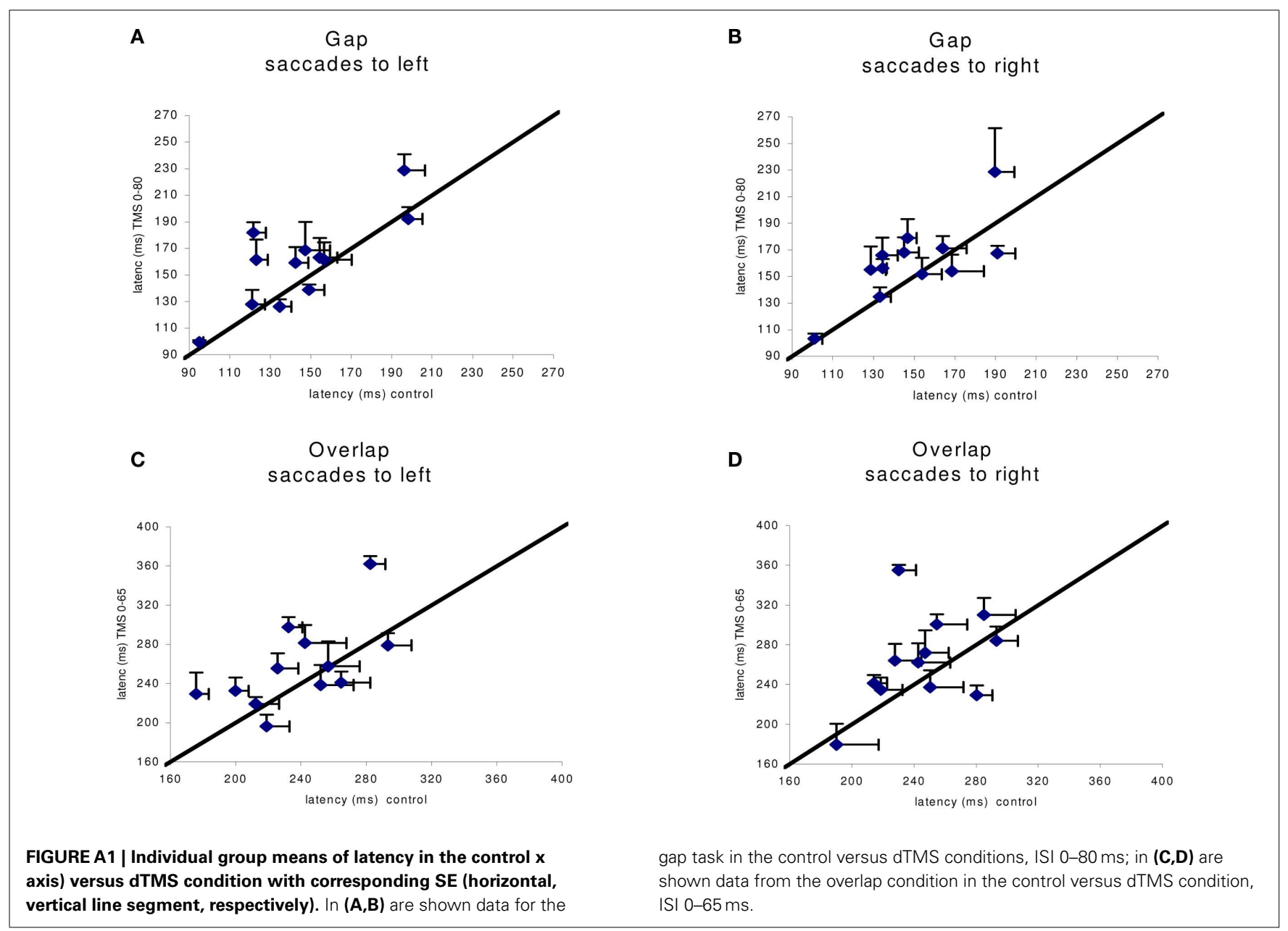

